\title{
On the Diversity, Multiplexing, and Array Gain Tradeoff in MIMO Channels
}

\author{
Luis G. Ordóñez, ${ }^{1}$ Daniel P. Palomar, ${ }^{2}$ and Javier R. Fonollosa ${ }^{1}$ \\ ${ }^{1}$ Signal Processing and Communications Group, Universitat Politècnica de Catalunya - Barcelona Tech \\ Jordi Girona 1-3, Campus Nord, Edifici D5, 08034 Barcelona (SPAIN) \\ ${ }^{2}$ Dept. of Electric and Computer Engineering, Hong Kong University of Science and Technology (HKUST) \\ Clear Water Bay, Kowloon, Hong Kong
}

\begin{abstract}
Following the seminal work of Zheng and Tse on the diversity and multiplexing tradeoff (DMT) of MIMO channels, in this paper we introduce the array gain to further investigate the fundamental relation between transmission rate and reliability in MIMO systems. The array gain gives information on the power offset that results from exploiting channel state information at the transmitter or, simply, because of the channel model. Hence, the diversity, multiplexing, and array gain (DMA) tradeoff is able to cope with the limitations of the original DMT and provide with operational meaning in the sense that the DMA tradeoff of a particular system can be directly translated into a parameterized characterization of its associated outage probability performance. As a first step towards this objective, we present in this paper the fundamental DMA tradeoff achievable by any scheme in uncorrelated Rayleigh block-fading MIMO channels.
\end{abstract}

\section{INTRODUCTION.}

\section{A. Benefits of MIMO Channels}

Multiple-input multiple-output (MIMO) channels are well know to provide a number of advantages over conventional single-antenna (SISO) channels, which have been traditionally described by the diversity, multiplexing, and array gain [1].

The diversity gain is the improvement in link reliability obtained by receiving replicas of the information signal through (ideally independent) fading links. With an increasing number of independent copies, the probability that at least one of the signals is not experiencing a deep fade increases, thereby improving the quality and reliability of reception.

The multiplexing gain is responsible for MIMO systems offering a linear increase in the achievable data rate. Indeed, in a MIMO channel, multiple independent data streams can be transmitted within the bandwidth of operation and, under suitable channel conditions, these can be separated at the receiver. Furthermore, each data stream experiences at least the same channel quality that would be experienced by a SISO system, effectively enhancing the capacity by a multiplicative factor equal to the number of established streams.

\footnotetext{
1 This work was partly funded by the Spanish Science and Technology Commissions and FEDER funds from the EC (TEC2006-06481/TCM and CONSOLIDER INGENIO CSD2008-00010 COMONSENS).

${ }^{2}$ This work was supported by the RGC 618008 research grant.
}

Finally, the array gain denotes the improvement in received signal-to-noise ratio (SNR) that results from a coherent combining effect of the information signals. The coherent combining may be realized through spatial processing at the receive antenna array and/or spatial pre-processing at the transmit antenna array.

\section{B. Fundamental Tradeoff}

Different design criteria of MIMO communication schemes are based on exploiting the previous gains, especially the spatial diversity and multiplexing gains. Actually, both perspectives come from different ways of understanding the ever-present fading in wireless communications. This dichotomic view of the fading process and by extension of the analysis and design MIMO systems is, however, not appropriate. In fact, given a MIMO channel, both the spatial diversity and the multiplexing gains can be simultaneously obtained, but there is a tradeoff between how much of each type of gain any MIMO scheme can extract: higher spatial multiplexing comes at the price of sacrificing diversity.

The complete picture of the diversity and multiplexing tradeoff (DMT) was given by Zheng and Tse in the excellent groundbreaking paper [2]. To be more specific, [2] focuses on the high-SNR regime and provides the fundamental tradeoff curve achievable by any scheme, where the spatial multiplexing gain is understood as the fraction of capacity attained at high SNR and the diversity gain indicates the high-SNR reliability of the system. The main problem, however, is that the DMT only provides a coarse measure of performance in the sense that it does not capture the impact of various relevant channel features and it is also insensitive to the presence of channel state information (CSI) at the transmitter [3]. Furthermore, it is difficult to translate any conclusion extracted from the DMT into the actual error probability of a particular scheme.

Several attempts have been made in the literature to provide the DMT with operational significance. First, we have the finite-SNR DMT derived in [4], where diversity and multiplexing gains definitions are modified to hold for any finite SNR value. However, the derivations are based on a lower 
bound on the outage probability and the final results require an additional numerical optimization process. Similarly, the finite-SNR DMT is also addressed in [5] for asymptotically large systems as either as the number of antennas in one or both sides of the link approaches infinity.

A totally different approach is taken in [6], where the focus is again in the high-SNR regime but the notion of multiplexing gain is substituted by that of rate region to investigate scenarios in which the data rate does not scale linearly with the logarithm of the SNR as in [2]. However, the throughput and reliability tradeoff in [6] is still independent of important parameters of the channel model.

\section{Contributions}

Here we aim at completing the DMT framework by including the array gain in the picture while trying to keep the essence of the original formulation. That is, we use equivalent definitions of diversity and multiplexing gain to those in [2] and include a new performance indicator which is able to cope with the limitations of the DMT. The array gain, indeed, gives information on the power offset that results from exploiting CSI at the transmitter or, simply, because of the adopted channel model. The resulting diversity, multiplexing, and array gain (DMA) tradeoff provides then more insights into the fundamental relation between transmission rate and reliability in MIMO systems, since the error probability is now characterized by two parameters: diversity and array gains. In this sense, the DMA tradeoff is still a two-fold tradeoff and must be not understood as a three-sided compromise between diversity, multiplexing, and array gains.

As a first step towards this objective, we present in this paper the fundamental DMA tradeoff achievable by any scheme in uncorrelated Rayleigh block-fading MIMO channels. The rest of the paper is organized as follows. In Section II the system and channel model are introduced. Then, the DMA tradeoff framework is presented in Section III and solved in Section IV. Finally, the main contribution of the paper is summarized in Section V.

\section{SYSTEM MODEL}

We consider a delay-limited wireless communication system with $n_{\mathrm{T}}$ transmit and $n_{\mathrm{R}}$ receive antennas, in which the channel matrix $\mathbf{H}$ remains constant within a block of $n_{\mathrm{S}}$ symbols. In this situation, the received signal within one block can be gathered in an $n_{\mathrm{R}} \times n_{\mathrm{S}}$ matrix $\mathbf{Y}$ related to the $n_{\mathrm{T}} \times n_{\mathrm{S}}$ transmitted matrix $\mathbf{X}$ as

$$
\mathbf{Y}=\mathbf{H X}+\mathbf{W}
$$

where $\mathbf{W}$ is the additive white Gaussian noise and has i.i.d. entries with zero mean and unit variance, and $\mathbf{H}$ follows an uncorrelated Rayleigh block-fading MIMO channel model, i.e., the entries of $\mathbf{H}$ are i.i.d. circularly symmetric Gaussian random variables with zero mean and unit variance that remain fixed for $n_{\mathrm{S}}$ channel uses after which they change to independent values. The transmitted signal $\mathbf{X}$ is normalized forcing the transmit power per block to satisfy

$$
\mathbb{E}\left\{\|\mathbf{X}\|_{\mathrm{F}}^{2}\right\} \leq n_{\mathrm{S}} \mathrm{snr}
$$

where snr is the average SNR at each receive antenna. The instantaneous CSI is assumed to be perfectly known at the receiver but may or may not be available at the transmitter.

Under such a system setup, the outage probability is the primary measure of interest in the sense that it is widely accepted to be the best achievable frame error probability (FER) in the limit of large codeword length, although the strong converse has been only proved for a restricted class of codes [7]. The outage probability is defined as the infimum of the probability that the instantaneous mutual information falls below the transmission rate $\mathrm{R}$ and, for the system model in (1), is given by [2, Sec. III.B]

$$
\mathrm{P}_{\text {out }}(\mathrm{R}, \text { snr })=\inf _{\mathbf{Q} \geq 0, \operatorname{tr}(\mathbf{Q}) \leq \text { snr }} \operatorname{Pr}\left(\log \left|\mathbf{I}_{n_{\mathrm{R}}}+\mathbf{H Q \mathbf { H } ^ { \dagger }}\right|<\mathbf{R}\right) .
$$

\section{DMA TRADEOFF FORMULATION}

Recall that our main objective is to derive a new framework based on the DMT able to provide additional information on the system performance by including the array gain. Hence, let us first formalize the definitions of diversity, multiplexing, and array gains.

As in [2], we define a scheme as a family of codes $\{\mathcal{C}($ snr $)\}$ of block length $n_{\mathrm{S}}$, which employs a different code $\mathcal{C}(\mathrm{snr})$ with rate $\mathrm{R}$ (snr) for each SNR level. Then, a MIMO coding scheme $\{\mathcal{C}($ snr $)\}$ is said to achieve a spatial multiplexing gain $r$, a diversity gain $d(r)$, and an array gain $a(r)$ if the data rate is such that

$$
\lim _{s n r \rightarrow \infty} \frac{R(s n r)}{\log s n r}=r
$$

and the outage probability satisfies

$$
\begin{aligned}
\lim _{\text {snr } \rightarrow \infty} \frac{\log P_{\text {out }}(r, s n r)}{\log s n r} & =-d(r) \\
\lim _{\text {snr } \rightarrow \infty} \frac{P_{\text {out }}(r, s n r)}{s n r^{-d}(r)} & =a(r)^{-d(r) .}
\end{aligned}
$$

The multiplexing gain definition coincides exactly with that in [2], while the diversity gain differs from that in [2] in the fact that we use the outage probability instead of the FER. However, for the fundamental DMA tradeoff addressed in this paper both definitions become equivalent, whenever the outage probability provides the best achievable FER.

Observe that definitions in (5) and (6) induce the following approximation of the high-SNR behavior of the outage probability when $\mathrm{R}$ satisfies (4):

$$
\mathrm{P}_{\text {out }}(\mathrm{r}, \mathrm{snr})=(\mathrm{a}(\mathrm{r}) \mathrm{snr})^{-\mathrm{d}(\mathrm{r})}+o\left(\mathrm{snr} \mathrm{r}^{-\mathrm{d}(\mathrm{r})}\right) \text {. }
$$

Hence, the DMA tradeoff of a particular system can be directly translated into a parameterized characterization of its associated outage probability performance. This enables the direct comparison of different strategies under different channel models and CSI assumptions. 


\section{DMA TRADEOFF IN MIMO CHANNELS}

In this section we derive the DMA tradeoff introduced in the previous section by obtaining the first order series expansion of the outage probability in (7). First, we distinguish between the case of having perfect CSI at both sides of the link (CSIT) and that of having CSI only available at the receiver (CSIR).

In principle, CSIT can be used to adjust the data rate to avoid channel outages. However, in delay-limited systems, the data rate is fixed and cannot depend on the channel variations except in outage states, where the channel does not support the desired rate and the data to be transmitted is lost. Under these circumstances, the outage probability is minimized with respect to the input distribution when waterfilling i.i.d. Gaussian codes along the channel eigenmodes [8, Prop. 3]:

$$
\mathrm{P}_{\text {out }}^{\text {(csit) }}(\mathrm{R}, \text { snr })=\operatorname{Pr}\left(\prod_{t=1}^{n}\left(1+p_{t}(\boldsymbol{\lambda}) \lambda_{t}\right)<2^{\mathrm{R}}\right) .
$$

where $n=\min \left(n_{\mathrm{T}}, n_{\mathrm{R}}\right), \lambda_{1} \geq \cdots \geq \lambda_{n} \geq 0$ denote the nonzero ordered eigenvalues of $\mathbf{H H}^{\dagger}$, and the power allocation is given by

$$
p_{t}(\boldsymbol{\lambda})=\left(\mu(\boldsymbol{\lambda})-\lambda_{t}^{-1}\right)^{+}
$$

where $(x)^{+}=\max (x, 0)$ and the water level $\mu(\boldsymbol{\lambda})$ is selected to satisfy the power constraint $\sum_{t=1}^{n} p_{t}(\boldsymbol{\lambda}) \leq \mathrm{snr}$ with equality.

Let us now concentrate on the high-SNR regime when the rate $\mathrm{R}$ satisfies (4). Using the same procedure as in the proof of [3, Thm. 2], it is not difficult to show that the outage probability in (8) is asymptotically equivalent to that achieved when allocating equal power to the $n$ available channel eigenmodes:

$$
\mathrm{P}_{\text {out }}^{(\mathrm{csit})}(\mathrm{r}, \text { snr }) \sim \mathrm{P}_{\text {out }}^{(\mathrm{n})}(\mathrm{r}, \mathrm{snr})
$$

where

$$
\mathrm{P}_{\text {out }}^{(\phi)}(\mathrm{r}, \mathrm{snr})=\operatorname{Pr}\left(\prod_{t=1}^{n}\left(1+\frac{\mathrm{snr}}{\phi} \lambda_{t}\right)<\mathrm{snr} r\right)
$$

being $\phi$ a strictly positive constant and ' $\sim$ ' denoting asymptotic equivalence as snr $\rightarrow \infty$, i.e., $f(\mathrm{snr}) \sim g(\mathrm{snr})$ if $\lim _{\mathrm{snr} \rightarrow \infty} f(\mathrm{snr}) / g(\mathrm{snr})=1$.

When only CSIR is available, the optimization of the input covariance matrix becomes more problematic and it has not even been solved for the canonical channel model we are considering. However, for this case, Telatar gives in [9] the widely accepted conjecture that the optimal strategy is to transmit i.i.d. codes with equal power through a subset $t$ out of the $n_{\top}$ antennas. In any case, it holds that [7]

$$
\mathrm{P}_{\text {out }}^{(\mathrm{csir})}(r, \mathrm{snr}) \leq \mathrm{P}_{\text {out }}^{\left(\mathrm{n}_{\mathrm{T}}\right)}(\mathrm{r}, \mathrm{snr}) .
$$

Since having CSI at the transmitter cannot penalize the outage probability, i.e., $\mathrm{P}_{\text {out }}^{\text {(csit) }}(r, s n r) \leq \mathrm{P}_{\text {out }}^{\text {(csir) }}(r, s n r)$, we can conclude that the upper-bound in (12) is asymptotically tight at least when $n=n_{\mathrm{T}}$. This fact has been already pointed out in [3] when analyzing the high-SNR outage capacity in block-fading MIMO channels.
Consequently, we restrict here to the case in which the covariance matrix $\mathbf{Q}$ is a scaled identity matrix, i.e.,

$$
\mathbf{Q}=\frac{\mathrm{snr}}{\phi} \mathbf{I}_{n_{\mathrm{T}}} \text {. }
$$

Then, the outage probability when $\mathrm{R}$ satisfies (4) is given by (11) and provides the high-SNR behavior of the outage probability under the CSIT and CSIR assumptions for $\phi=n$ and $\phi=n_{\mathrm{T}}$, respectively. The resulting DMA tradeoff under uncorrelated Rayleigh fading is presented in the next theorem.

Theorem 1: The outage probability in an uncorrelated Rayleigh fading $n_{\mathrm{T}} \times n_{\mathrm{R}}$ MIMO channel when $\mathrm{R}$ satisfies (4) and the covariance matrix is restricted to be as in (13) is

$$
\mathrm{P}_{\text {out }}^{(\phi)}(\mathrm{r}, \mathrm{snr})=\left(\mathrm{a}^{(\phi)}(\mathrm{r}) \mathrm{snr}\right)^{-\mathrm{d}(\mathrm{r})}+o\left(\mathrm{snr}^{-\mathrm{d}(\mathrm{r})}\right)
$$

where:

(i) The multiplexing gain is denoted by $r$.

(ii) The diversity gain $\mathrm{d}(\mathrm{r})$ for $0 \leq \mathrm{r} \leq n$ is given by

$$
\mathrm{d}(\mathrm{r})=G_{\mathrm{d}}(k)-G_{\mathrm{r}}(k) \mathrm{r}
$$

where $k=\lfloor r\rfloor=\{k \in \mathbb{N} \mid k \leq r\}$, and

$$
\begin{aligned}
& G_{\mathrm{d}}(k)=m n-k(k+1) \\
& G_{\mathrm{r}}(k)=m+n-(2 k+1)
\end{aligned}
$$

with $n=\min \left(n_{\mathrm{T}}, n_{\mathrm{R}}\right)$ and $m=\max \left(n_{\mathrm{T}}, n_{\mathrm{R}}\right)$.

(iii) The array gain $\mathrm{a}^{(\phi)}(\mathrm{r})$ for $0<\mathrm{r}<n$ is given by

$\mathrm{a}^{(\phi)}(\mathrm{r})=\left(K_{m, n}\left|\mathbf{K}_{m, n}(k)\right|\left(\prod_{t=1}^{k}(t-1) ! t !\right)\left(\frac{\phi^{G_{\mathrm{d}}(k)}}{G_{\mathrm{r}}(k)}\right)\right)^{-1 / \mathrm{d}(\mathrm{r})}$

where

$$
K_{m, n}=\prod_{t=1}^{n} \frac{1}{(n-t) !(m-t) !}
$$

and matrix $\mathbf{K}_{m, n}(k)$ is defined as

$$
\left[\mathbf{K}_{m, n}(k)\right]_{u, v}=\sum_{i=0}^{m-n}\left(\begin{array}{c}
m-n \\
i
\end{array}\right) \frac{(-1)^{i}}{(u+v+i)}
$$

for $u, v=1, \ldots, n-k-1$.

Remark 1: Observe that the DMT tradeoff curve in [2, Thm. 1] can be recovered from Theorem 1 as

$$
d(\mathrm{r})=-\lim _{\text {snr } \rightarrow \infty} \frac{\log \mathrm{P}_{\text {out }}^{(\phi)}(\mathrm{r}, \mathrm{snr})}{\log \mathrm{snr}}=G_{\mathrm{d}}(k)-G_{\mathrm{r}}(k) \mathrm{r}
$$

where $G_{\mathrm{d}}(k)$ and $G_{\mathrm{r}}(k)$ are given in (16) and (17), respectively.

Remark 2: For $m=n$, the determinant $\left|\mathbf{K}_{m, n}(k)\right|$ in (18) can be evaluated in closed form using Cauchy's double alternant [10, eq. (2.7)]

$$
\begin{aligned}
\left|\mathbf{K}_{m, n}(k)\right| & =\sum_{\boldsymbol{\mu}} \operatorname{sgn}(\boldsymbol{\mu}) \prod_{t=1}^{n-k-1} \frac{1}{\left(\mu_{t}+t+i\right)} \\
& =\frac{\prod_{u<v}^{n-k-1}(u-v)^{2}}{\prod_{u=1}^{n-k-1} \prod_{v=1}^{n-k-1}(u+v+i)} .
\end{aligned}
$$


Proof of Theorem 1: Under uncorrelated Rayleigh fading, either $\mathbf{H H}^{\dagger}$ or $\mathbf{H}^{\dagger} \mathbf{H}$ is uncorrelated central Wishart distributed [11, Sec. II]. Since the non-zero ordered eigenvalues of $\mathbf{H H}^{\dagger}$ and $\mathbf{H}^{\dagger} \mathbf{H}$ coincide, the joint pdf of $\boldsymbol{\lambda}=\left(\lambda_{1}, \ldots, \lambda_{n}\right)$ is given by $[12$, eq. (95)]

$$
f_{\boldsymbol{\lambda}}(\boldsymbol{\lambda})=K_{m, n} \prod_{i<j}^{n}\left(\lambda_{j}-\lambda_{i}\right)^{2} \prod_{i=1}^{n} e^{-\lambda_{i}} \lambda_{i}^{m-n}
$$

where $K_{m, n}$ is defined in (19). Let us now introduce the ordered variables $\alpha_{1} \geq \cdots \geq \alpha_{n} \geq 1$ with

$$
\alpha_{t}=1+\frac{\mathrm{snr}}{\phi} \lambda_{t} \quad \text { for } t=1, \ldots, n
$$

so that the outage probability in (11) can be rewritten in terms of $\boldsymbol{\alpha}=\left(\alpha_{1}, \ldots, \alpha_{n}\right)$ as

$$
\mathrm{P}_{\text {out }}^{(\phi)}(\mathrm{r}, \mathrm{snr})=\operatorname{Pr}\left(\prod_{t=1}^{n} \alpha_{t}<\mathrm{snr}^{\mathrm{r}}\right)=\int_{\mathcal{A}} f_{\boldsymbol{\alpha}}(\boldsymbol{\alpha}) d \boldsymbol{\alpha}
$$

where $\mathcal{A}=\left\{\boldsymbol{\alpha} \in \mathbb{R}^{n} \mid \prod_{t=1}^{n} \alpha_{t}<\right.$ snr $^{\mathrm{r}}$ and $\alpha_{1} \geq \cdots \geq \alpha_{n} \geq$ $1\}$ and $f_{\boldsymbol{\alpha}}(\boldsymbol{\alpha})$ denotes the joint pdf of $\boldsymbol{\alpha}$ given by

$$
\begin{aligned}
f_{\boldsymbol{\alpha}}(\boldsymbol{\alpha}) & =\left(\frac{\phi}{\mathrm{snr}}\right)^{n} f_{\boldsymbol{\lambda}}\left(\left(\frac{\phi}{\mathrm{snr}}\right)\left(\alpha_{1}-1\right), \ldots,\left(\frac{\phi}{\mathrm{snr}}\right)\left(\alpha_{n}-1\right)\right) \\
& =K_{m, n}\left(\frac{\phi}{\mathrm{snr}}\right)^{m n} e^{n\left(\frac{\phi}{\mathrm{snr}}\right)} \prod_{i<j}^{n}\left(\alpha_{j}-\alpha_{i}\right)^{2} \\
& \prod_{i=1}^{n} e^{-\left(\frac{\phi}{\mathrm{snr}}\right) \alpha_{i}}\left(\alpha_{i}-1\right)^{m-n} \cdot(27
\end{aligned}
$$

In order to obtain (14), it is important to observe first that not all $\alpha \in \mathcal{A}$ contribute to the high-SNR behavior of $\mathrm{P}_{\text {out }}^{(\phi)}(r$, snr $)$ in (26). This can be easily investigated using Laplace's Principle [13, Ch. 5]. However, we already know from the proof of [2, Thm. 1] that the term with highest SNR exponent comes from integrating $f_{\boldsymbol{\alpha}}(\boldsymbol{\alpha})$ in a neighborhood of points $\boldsymbol{\alpha}$ satisfying

$$
\log _{\text {snr }}\left(\alpha_{t}\right)= \begin{cases}1 & 1 \leq t \leq k \\ r-k & t=k+1 \\ 0 & k+1<t \leq n\end{cases}
$$

where $k=\lfloor r\rfloor$. When $\boldsymbol{\alpha}$ satisfies (28), the following asymptotic equivalences hold:

$$
\begin{aligned}
& \prod_{i=1}^{k} \prod_{j=k+1}^{n}\left(\alpha_{j}-\alpha_{i}\right)^{2} \sim \prod_{t=1}^{k} \alpha_{t}^{2(n-k)} \\
& \prod_{t=1}^{k}\left(\alpha_{t}-1\right)^{m-n} \sim \prod_{t=1}^{k} \alpha_{t}^{m-n} \\
& e^{n\left(\frac{\phi}{\mathrm{snr}}\right)} \sim e^{-\left(\frac{\phi}{\mathrm{snr}}\right) \alpha_{k+1}} \sim \cdots \sim e^{-\left(\frac{\phi}{\mathrm{snr}}\right) \alpha_{n}} \sim 1 .
\end{aligned}
$$

Noting that

$$
\begin{aligned}
\prod_{i<j}^{n}\left(\alpha_{j}-\alpha_{i}\right)^{2}= & \prod_{i=1}^{k} \prod_{j=k+1}^{n}\left(\alpha_{j}-\alpha_{i}\right)^{2} \\
& \prod_{i<j}^{k}\left(\alpha_{j}-\alpha_{i}\right)^{2} \prod_{k<i<j}^{n}\left(\alpha_{j}-\alpha_{i}\right)^{2}
\end{aligned}
$$

and taking into account previous considerations, the high-SNR behavior of $\mathrm{P}_{\text {out }}^{(\phi)}(r, s n r)$ in (26) is given by

$$
\begin{aligned}
& \mathrm{P}_{\text {out }}^{(\phi)}(\mathrm{r}, \mathrm{snr}) \sim K_{m, n}\left(\frac{\phi}{\mathrm{snr}}\right)^{m n} \int_{\mathcal{A}} \prod_{t=1}^{k} e^{-\left(\frac{\phi}{\mathrm{snr}}\right) \alpha_{t}} \alpha_{t}^{\zeta(2 k)} \\
& \prod_{i<j}^{k}\left(\alpha_{j}-\alpha_{i}\right)^{2} \prod_{k<i<j}^{n}\left(\alpha_{j}-\alpha_{i}\right)^{2} \prod_{t=k+1}^{n}\left(\alpha_{t}-1\right)^{m-n} d \boldsymbol{\alpha}
\end{aligned}
$$

where $\zeta(t)=m+n-t$. Let us now define

$$
\begin{aligned}
A_{(k)}(\mathrm{r}, \mathrm{snr}) & =\int_{\mathcal{A}_{(k)}} \prod_{t=1}^{k} e^{-\left(\frac{\phi}{\mathrm{snr}}\right) \alpha_{t}} \alpha_{t}^{\zeta(2 k)} \\
& \prod_{i<j}^{k}\left(\alpha_{j}-\alpha_{i}\right)^{2} A^{(k)}\left(\alpha_{1}, \ldots, \alpha_{k}\right) d \alpha_{k} \cdots d \alpha_{1}
\end{aligned}
$$

where $\mathcal{A}_{(k)}=\left\{\left(\alpha_{1}, \ldots, \alpha_{k}\right) \in \mathbb{R}^{k} \mid \prod_{t=1}^{k} \alpha_{t}<\right.$ snr $^{r}$ and $\left.\alpha_{1} \geq \cdots \geq \alpha_{k} \geq 1\right\}$, and

$$
\begin{aligned}
A^{(k)}\left(\alpha_{1}, \ldots, \alpha_{k}\right)= & \int_{\mathcal{A}^{(k)}} \prod_{k<i<j}^{n}\left(\alpha_{j}-\alpha_{i}\right)^{2} \\
& \prod_{t=k+1}^{n}\left(\alpha_{t}-1\right)^{m-n} d \alpha_{n} \cdots d \alpha_{k+1}
\end{aligned}
$$

where $\mathcal{A}^{(k)}=\mathcal{A}^{(k)}\left(\alpha_{1}, \ldots, \alpha_{k}\right)=\left\{\left(\alpha_{k+1}, \ldots, \alpha_{n}\right) \in\right.$ $\mathbb{R}^{n-k} \mid \prod_{t=1}^{n} \alpha_{t}<\operatorname{snr}^{r}$ and $\left.\alpha_{k+1} \geq \cdots \geq \alpha_{n} \geq 1\right\}$. Then, since $\alpha_{k} \geq \alpha_{k+1}$ is guaranteed for any $\boldsymbol{\alpha}$ satisfying (28), integrating over $\mathcal{A}^{(k)}$ and subsequently over $\mathcal{A}_{(k)}$ is equivalent to integrating directly over $\mathcal{A}$. Hence, we can rewrite (33) as

$$
\mathrm{P}_{\text {out }}^{(\phi)}(\mathrm{r}, \mathrm{snr}) \sim K_{m, n}\left(\frac{\phi}{\mathrm{snr}}\right)^{m n} A_{(k)}(\mathrm{r}, \mathrm{snr}) .
$$

The integral $A^{(k)}\left(\alpha_{1}, \ldots, \alpha_{k}\right)$ in (35) is shown in [14] to satisfy

$$
A^{(k)}\left(\alpha_{1}, \ldots, \alpha_{k}\right) \sim \frac{1}{\zeta(2 k+1)}\left|\mathbf{K}_{m, n}(k)\right|\left(\frac{\operatorname{snr}^{r}}{\prod_{t=1}^{k} \alpha_{t}}\right)^{\zeta(2 k+1)}
$$

where matrix $\mathbf{K}_{m, n}(k)$ is defined in (20). Then, the asymptotic behavior of $A_{(k)}(r$, snr), when substituting (37) back in (34), is shown in [14] to be given by

$$
\begin{aligned}
A_{(k)}(\mathrm{r}, \mathrm{snr}) \sim\left|\mathbf{K}_{m, n}(k)\right| & \left(\prod_{t=1}^{k}(t-1) ! t !\right) \\
& \left(\frac{\operatorname{snr}^{\mathrm{r} \zeta(2 k+1)}}{\zeta(2 k+1)}\right)\left(\frac{\mathrm{snr}}{\phi}\right)^{k(k+1)} .
\end{aligned}
$$

The expressions in (37) and (38) come from identifying $\prod_{k<i<j}^{n}\left(\alpha_{j}-\alpha_{i}\right)$ and $\prod_{i<j}^{k}\left(\alpha_{j}-\alpha_{i}\right)$ as Vandermonde determinants [15, eq. (6.1.33)] and exploiting the symmetry of both integrands to calculate the integrals over $\mathcal{A}^{(k)}$ and $\mathcal{A}_{(k)}$ in the corresponding unordered domains as in [11, App. B.2].

Finally, combining (38) with (36), the first order series expansion of $\mathrm{P}_{\text {out }}^{(\phi)}(r, \mathrm{snr})$ given in the theorem follows. 


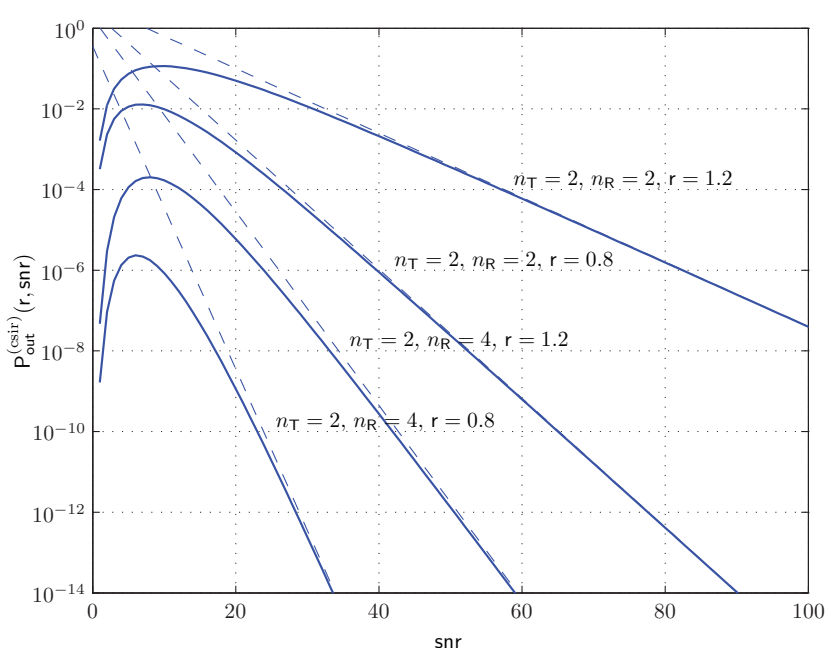

Fig. 1. Simulated outage probability (solid) and DMA tradeoff (dashed).

Theorem 1 holds only for the uncorrelated Rayleigh MIMO channel, however, the extension to other common channel models such as correlated Rayleigh or Ricean MIMO channels is not difficult. It is well known that all these channels have the same DMT [16] and, hence, only the array gain must be obtained. Since the joint pdf of the ordered eigenvalues of these channel models share a common structure [11], similar techniques as in the proof of Theorem 1 can be applied.

Finally, for illustrative purposes we show in Figures 1 and 2 the simulated outage probability and the high-SNR behavior derived from the DMA tradeoff under uncorrelated Rayleigh fading and no CSI knowledge at the transmitter when imposing an isotropic input covariance matrix. Since the target data rate is chosen as $R=r \log s n r$ and, the simulated outage probability is not representative for low SNR values. The remaining part of the outage probability curve is, as expected, well approximated by the DMA tradeoff in Theorem 1. We emphasize that the DMT provides the slopes of the curves but not the horizontal shift. This is precisely the contribution of the present paper.

\section{CONCLUSIONS}

Zheng and Tse 2003 paper was the first one to reveal and quantify the fundamental interconnection present in MIMO channels between the multiplexing gain, associated to rate, and the diversity gain, related to the slope of the error rate. This characterization is however difficult to be translated into practical performance indicators like frame error rate without the addition of a third parameter, the array gain, that provides a constant shift to the diversity gain slope resulting in an asymptotic affine characterization of the error curve in the logarithmic domain. This paper introduces the diversity, multiplexing, and array gain tradeoff opening the door for a more illustrative performance evaluation of MIMO schemes.

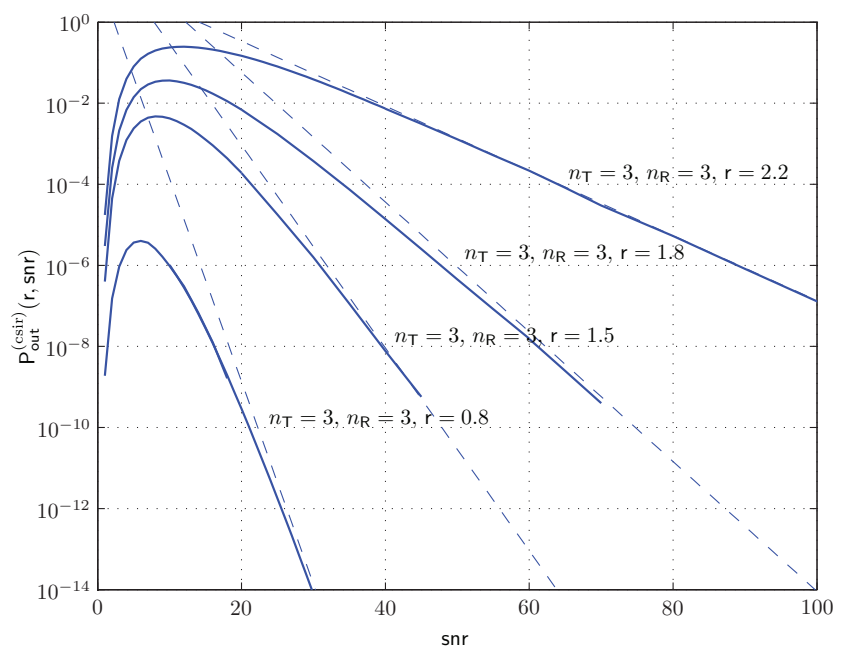

Fig. 2. Simulated outage probability (solid) and DMA tradeoff (dashed).

\section{REFERENCES}

[1] H. Bölcskei and A. J. Paulraj, "Multiple-input multiple-output (MIMO) wireless systems," in The Communications Handbook, 2nd ed. CRC Press, 2002.

[2] L. Zheng and D. N. C. Tse, "Diversity and multiplexing: a fundamental tradeoff in multiple-antenna channels," IEEE Trans. Inform. Theory, vol. 49, no. 5, pp. 1073-1096, May 2003.

[3] N. Prasad and M. K. Varanasi, "MIMO outage capacity in the high SNR regime," Proc. IEEE Int. Symp. Inform. Theory (ISIT), pp. 656 - 660, 2005.

[4] R. Narasimhan, "Finite-SNR diversity-multiplexing tradeoff for correlated Rayleigh and Rician MIMO channels," IEEE Trans. Inf. Theory, vol. 52, no. 9, pp. 3965-3979, Sep. 2006.

[5] S. Loyka and G. Levin, "Diversity-multiplexing tradeoff via asymptotic analysis of large systems," Proc. IEEE Int. Symp. Inform. Theory (ISIT), 2007.

[6] K. Azarian and H. E. Gamal, "The throughput-reliability tradeoff in block-fading MIMO channels," IEEE Trans. Inf. Theory, vol. 53, no. 2, pp. $488-501$, Feb. 2007

[7] N. Prasad and M. K. Varanasi, "Outage theorems for MIMO blockfading channels," IEEE Trans. Inf. Theory, vol. 52, no. 12, pp. 52845296, Dec. 2006.

[8] E. Biglieri, G. Taricco, and A. M. Tulino, "Performance of space-time codes for a large number of antennas," IEEE Trans. Inform. Theory, vol. 48, no. 7, pp. 1794-1803, July 2002.

[9] I. E. Telatar, "Capacity of multi-antenna Gaussian channel," European Trans. Telecomm., vol. 10, no. 6, pp. 585-595, Nov./Dec. 1999.

[10] C. Krattenthaler, "Advanced determinant calculus," Seminaire Lotharingien Combin. 42 ("The Andrews Festschrift"), 1999.

[11] L. G. Ordóñez, D. P. Palomar, and J. R. Fonollosa, "Ordered eigenvalues of a general class of Hermitian random matrices with application to the performance analysis of MIMO systems," IEEE Trans. Signal Processing, vol. 57, no. 2, pp. 672-689, Feb. 2009.

[12] A. T. James, "Distributions of matrix variates and latent roots derived from normal samples," Ann. of Math. Statist., vol. 35, no. 2, pp. 475501, Jun. 1964.

[13] K. W. Breitung, Asymptotic Approximations for Probability Integrals, ser. Lecture Notes in Mathematics. Springer-Verlag, 1994.

[14] L. G. Ordóñez, D. P. Palomar, and J. R. Fonollosa, "On the diversity, multiplexing, and array gain tradeoff in MIMO channels," Submitted to IEEE Trans. Inform. Theory, 2010.

[15] R. A. Horn and C. R. Johnson, Topics in Matrix Analysis. New York: Cambridge University Press, 1991.

[16] L. Zhao, W. Mo, Y. Ma, and Z. Wang, "Diversity and multiplexing tradeoff in general fading channels," IEEE Trans. Inf. Theory, vol. 53, no. 4, pp. 1549-1557, Apr. 2007. 